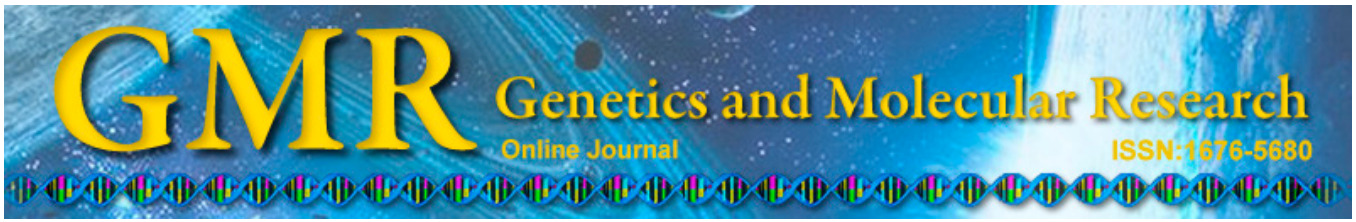

\title{
Effect of nimodipine on rat spinal cord injury
}

\author{
Y.-F. Jia, H.-L. Gao, L.-J. Ma and J. Li \\ Department of Neurosurgery, Affiliated Hospital of Yanan University, \\ Yanan, China \\ Corresponding author: Y.-F. Jia \\ E-mail: jiayun_feng123@163.com \\ Genet. Mol. Res. 14 (1): 1269-1276 (2015) \\ Received February 27, 2014 \\ Accepted July 28, 2014 \\ Published February 13, 2015 \\ DOI http://dx.doi.org/10.4238/2015.February.13.5
}

\begin{abstract}
We evaluated the potentially protective effect of nimodipine on rat spinal cord injury. Sprague-Dawley rats received spinal cord injury, and were separated into nimodipine $(\mathrm{N}=12)$ and saline groups $(\mathrm{N}=12)$. Within $1 \mathrm{~h}$ of the injury, rats were treated intraperitoneally with nimodipine $(1.0 \mathrm{mg} / \mathrm{kg})$ or an equal amount of saline. Treatment was performed 3 times a day for 1 week. Operation BBB score and track experiment were used to measure the physical function of the hind legs 1 and 2 weeks after injury. Two weeks after the injury, malondialdehyde (MDA) content and spinal cord myeloperoxidase (MPO) activity of the injured part were determined, and the glial scar and dead room were studied using the immune tissue chemical test. ED1 was used to observe active gitter cell and macrophages. The physical function of the nimodipine group improved significantly $(\mathrm{P}<0.01)$. Two weeks after injury, spinal cord MDA content in the spinal cord in the nimodipine group (nmol/g, $25.6 \pm$ 9.7 vs $68.5 \pm 16.7)$ and MPO activity $(\mathrm{U} / \mathrm{g}, 252.2 \pm 63.9$ vs $382.8 \pm$ 108.2) decreased significantly $(\mathrm{P}<0.01)$; nimodipine whole dead space $\left(\mathrm{mm}^{2}, 4.45 \pm 1.28\right.$ vs $\left.6.16 \pm 2.65\right)$ and ED1 antibody immunity colored positive room $\left(\mathrm{mm}^{2}, 1.87 \pm 0.42\right.$ vs $\left.2.86 \pm 1.01\right)$ reduced significantly
\end{abstract}


$(\mathrm{P}<0.01)$. Nimodipine treatment could reduce oxidative injury after spinal cord injury, reduce the whole dead space and inflammation, and repair spinal cord injury.

Key words: Rats; Spinal cord injury; Nimodipine; Propylene glycol; Peroxidase; Free radical

\section{INTRODUCTION}

Spinal cord injury (SCI) is often associated with obvious secondary pathological changes such as ischemia, free radical damage, inflammation, excitotoxic neuronal degeneration and necrosis or apoptosis (Zhang et al., 2012; Lang et al., 2012; Atılgan, 2012). Following the initial spinal cord injury, free radical damage caused by biochemical changes associated with lipid peroxidation are thought to cause additional deterioration of the original area of damage (Hawryluk et al., 2008; Ouardouz et al., 2009; Lockman et al., 2012). Biological markers of lipid peroxidation include MDA (malondialdehyde), a major end-product of polyunsaturated fatty acid peroxidation found in cell membranes (Ouardouz et al., 2009). The early inflammatory response involves extensive accumulation of inflammatory cells and cytokines and the secretion of large amounts of toxic free radicals, thereby increasing the extent of spinal cord injury; however, secretion of protective cytokines and growth factors, which mediate neuroprotection and promote nerve regeneration, also occurs in response to spinal cord injury (Hawryluk et al., 2008; Doukas et al., 2011; Lockman et al., 2012).

Nimodipine is a dihydropyridine calcium antagonist, which acts to selectively enhance brain blood flow without affecting cerebral metabolism, and which is widely used in pharmaceutical research and clinical treatment for spinal cord injury (Tan et al., 2005; Doukas et al., 2011; Han et al., 2011). In this study, adult Sprague-Dawley rats were used in a model of moderate impact spinal cord injury and the effects of early treatment with nimodipine on inflammatory responses at the site of injury were evaluated.

\section{MATERIAL AND METHODS}

\section{Animals}

Twenty-four healthy adult male Sprague-Dawley rats provided by the 4th Military Medical University Experimental Animal Center were used in this study. Subjects weighed 180-220 g, and were maintained under conditions of constant ventilation, 55\% humidity, and $22^{\circ} \mathrm{C}$ temperature. Lights remained on in the housing room day and night and the cages were regularly cleaned and disinfected. The rats were randomly divided into a nimodipine group $(\mathrm{N}=12)$ and a saline control group $(\mathrm{N}=12)$. The nimodipine used in this study was produced by Tianjin Central Pharmaceutical Co., Ltd. (permission No. H20043915).

\section{Procedure}

\section{SCI model}

The model of SCI used in this study is based on previous studies (Gruner, 1992; Nara- 
zaki et al., 2006; Zhou et al., 2008). We anesthetized the rats with sodium pentobarbital (0.5 $\mathrm{mg} / \mathrm{kg}$ ) and shaved the back, using Anerdian $75 \%$ alcohol after initial disinfection with iodine. The T8 spinal level was selected as the center for our $5 \mathrm{~cm}$ incision, after which we separated the subcutaneous tissue and muscle to expose and cut off the T8 spinal processes and perform a laminectomy exposing the dura. Using a computer, we controlled and recorded the impact velocity and compression values administered by an NYU (New York University) impact device (NYU impactor II). Our settings (10 g, $12.5 \mathrm{~cm}$ drop) resulted in a moderate spinal cord injury. The wound was sutured postoperatively and the rats' bladders were compressed 3 times/day to ensure sufficient urination.

\section{Medical treatment}

Within $1 \mathrm{~h}$ of injury, we began intraperitoneal administration of either nimodipine (nimodipine group, $1.0 \mathrm{mg} / \mathrm{kg}$ ) or an equivalent amount of normal saline (saline group), 3 times per day for 1 week.

\section{Assessment of hind limb motor function}

We conducted open field and track tests 1 and 2 weeks after injury to compare the motor function of the nimodipine- and saline-treated groups. In the open field test, we used the BBB score (Basso, Beattie \& Bresnahan locomotor rating scale) to assess spinal cord function after injury. In addition, 2 weeks after injury, we colored the plantar and dorsal surfaces of the rats' feet with different pigments and let them climb smooth planks covered with white paper (100 $\mathrm{x} 7 \mathrm{~cm}$ ), to assess their motor function recovery by analyzing their footprints. Specifically, we observed indicators of motor function which included step (distance between the two ipsilateral adjacent footprints) and step width (the distance between two adjacent right and left footprints).

\section{Malondialdehyde (MDA) and spinal cord peroxidase (MPO) activity}

MDA test: As a major end-product, the volume of MDA can reflect the degree of lipid peroxidation. The reaction principle is that MDA can react with thio barbituric acid (TBA) and condensated red products. A 48T/96T rats MDA kit was purchased from Shanghai Institute of Biological Technology Co., Ltd. and was used according to manufacturer instructions. We recorded the absorbance (OD) at a wavelength of $450 \mathrm{~mm}$ and took 4-ethoxypropane as the external standard, to calculate the amount of MDA expressed by means of the standard curve $(\mathrm{nmol} / \mathrm{g})$.

MPO test: The spinal cord MPO ELISA kit was purchased from Shanghai Yili Biotech Co, Ltd. With the MPO and hydrogen donor o-dianisidine to produce yellow complexes then test the MPO activity (U/g) by standard curve (Narazaki et al., 2006).

\section{Immunohistochemical examination}

Two weeks after the operation, the rats were killed and perfused with cold saline and $10 \%$ paraformaldehyde buffer through the heart. Subsequently, $2 \mathrm{~cm}$ of the spinal cord was fixed for $2 \mathrm{~h}$ with $10 \%$ paraformaldehyde buffer and embedded in $30 \%$ sucrose solution, and frozen. The constant cold box slicer (Leica, CA1900) was used to obtain sagittal serial sec- 
tions $(20 \mu \mathrm{m})$. In these serial sections, with the central tube as the center, on both sides of the interval of $200 \mu \mathrm{m}$ and $400 \mu \mathrm{m}$ for each selected slice and fluorescent immunohistochemistry, and finally with rabbit-derived GFAP (1:3000, Sigma-Aldrich, USA) labeled astrocytes scar, murine ED1/CD68 (1:500, Serotec, USA) mark active microglia and macrophages after spinal cord injury. We observed the microglial activation and macrophage infiltration. Then we used Photoshop CS2 to measure the void area $\left(\mathrm{mm}^{2}\right)$, Image ProPlus 6.0 (IPP6.0) to measure positive staining area $\left(\mathrm{mm}^{2}\right)$ from the midpoint of the range ED1. Then we used a fluorescence microscope (BX51, Olympus) cell changes observed slices and collected picture.

\section{Data analysis}

We used SPSS 16.0 to analyze our behavioral and biochemical data using analysis of variance. All values are reported as means \pm standard deviation $( \pm \mathrm{SD}) . \mathrm{P}<0.05$ indicates a significant difference.

\section{RESULTS}

\section{Motor function}

\section{Open field test (BBB score)}

Prior to SCI, the average BBB score of the rats was 21. After SCI, both groups showed reduced BBB scores, but motor function of the hind limbs of rats in the nimodipine group was significantly improved compared to that of the saline group ( $1 \mathrm{w}: 11.02 \pm 0.98$ vs $8.15 \pm 1 ., t=4.381, \mathrm{P}<0.01 ; 2 \mathrm{w}: 14.16 \pm 1.32$ vs $10.93 \pm 0.89, t=4.193, \mathrm{P}<0.01)$.

\section{Track test}

After $14 \mathrm{~d}$ of injury, there were signs of hind limb motor dysfunction, which included smaller step sizes and increased step width. Compared to the saline group, step size of the nimodipine group $(\mathrm{cm})$ increased $(11.77 \pm 0.92 v s 8.63 \pm 1.17, t=4.012, \mathrm{P}<0.01)$, and step width $(\mathrm{cm})$ became smaller $(4.06 \pm 0.42$ vs $5.28 \pm 0.86, t=3.976, \mathrm{P}<0.01)$.

\section{Changes of MDA and MPO}

Twenty-four h after injury, MDA (nmol/g, $20.7 \pm 8.5 v s 165.4 \pm 66.8, t=9.938, \quad \mathrm{P}<$ $0.01)$ and MPO activity $(\mathrm{U} / \mathrm{g}, 186.8 \pm 73.2$ vs $285.7 \pm 118.3, t=8.362, \mathrm{P}<0.01)$ were both increased. After 2 weeks of treatment, the nimodipine group showed significantly decreased expression of MDA (nmol/g) of nimodipine group (25.6 $\pm 9.7 v s 68.5 \pm 16.7, t=6.893, \mathrm{P}<$ $0.01)$ and $\mathrm{M} P O(\mathrm{U} / \mathrm{g})$ activity $(252.2 \pm 63.9 v s 382.8 \pm 108.2, t=7.152, \mathrm{P}<0.01)$ as compared to the saline group.

\section{Immunohistochemical changes}

Necrosis can be seen around the SCI site. There were signs of inflammatory cell infiltration and astrocyte proliferation, but part of the normal white matter structures remained 
as well. Fourteen days after injury, both the hollow area of the injury site $\left(\mathrm{mm}^{2}, 4.45 \pm 1.28\right.$ vs $6.16 \pm 2.65, t=4.015, \mathrm{P}<0.01$, Figure 1) and the area positive for ED1 antibody immunohistochemical staining $\left(\mathrm{mm}^{2}, 1.87 \pm 0.42 v s 2.86 \pm 1.01, t=3.892, \mathrm{P}<0.01\right.$, Figure 2$)$ were reduced significantly in the nimodipine group compared to the saline group.

A

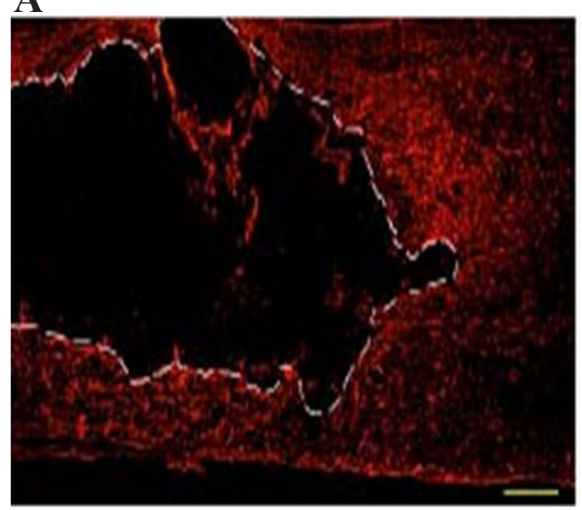

B

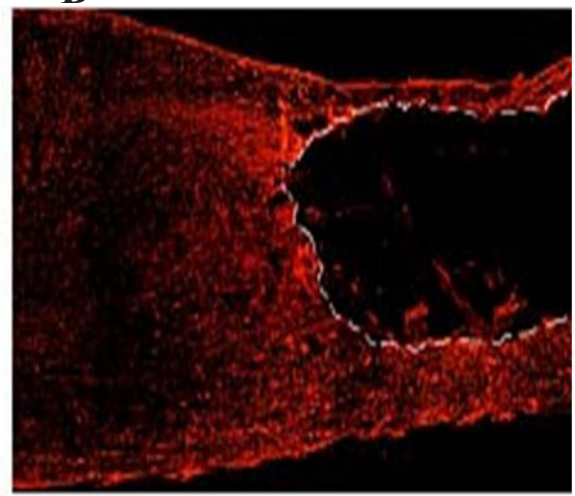

Figure 1. Area of spinal cord damage 2 weeks postoperatively, outlined by GFAP immunohistochemical staining (scale bar $=200 \mu \mathrm{m})$. A. Nimodipine group; B. normal saline group.

A

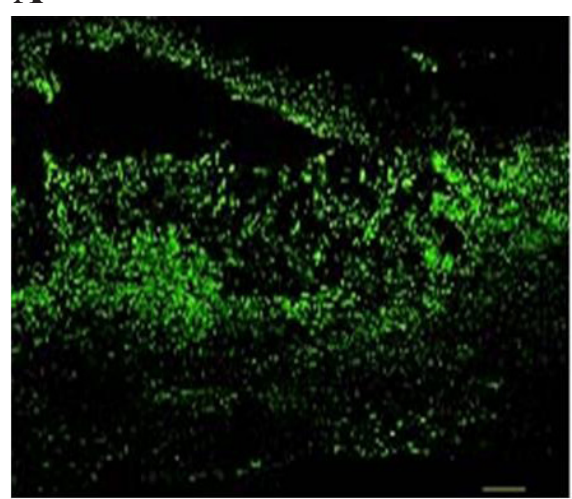

B

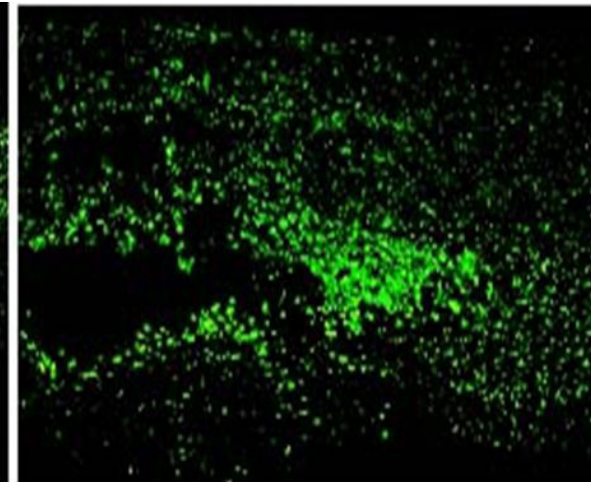

Figure 2. Area of spinal cord damage 2 weeks postoperatively, positive for ED1 antibody immunohistochemical staining (scale bar $=200 \mu \mathrm{m}$ ). A. Nimodipine group; B. normal saline group.

\section{DISCUSSION}

SCI often leads to severe motor dysfunction. In its most extreme form, it can result in complete paralysis, whereby patients may lose not only the ability to walk, but may also lose normal bowl, bladder, and sexual function. SCI pathophysiology is very complex, involving histopathological, electrophysiological and chemical changes in affected cells and tissues (Gonzalez et al., 2003; Wang et al., 2009; Hakan et al., 2011; Zhang et al., 2012; Lang et al., 2012; Attlgan, 2012). In order to clarify the complex mechanisms involved in SCI, and evaluate potential interventions in the treatment of SCI, it is important to use a model of SCI model 
which has high reliability and replicability (Zhao et al., 2011; Kumar et al., 2011; Vinit, 2012).

A variety of pathogenic factors can cause spinal cord injury, resulting in a range of pathological changes and dysfunctions (Vinit, 2012; Krause et al., 2012; Wijesuriya et al., 2012). One approach to treatment, stem cell therapy and rehabilitation of spinal cord injury, has achieved good results (Saunders et al., 2012; Lin et al., 2012; Chittiboina et al., 2012). Potential cellular mechanisms of these beneficial effects include the following: 1) after transplantation into the patient's body, stem cells can differentiate into neurons, astrocytes and oligodendrocytes, and produce a variety of extracellular matrix molecules, thus filling the cavity left after spinal cord injury with materials to support axonal regeneration, 2) stem cells transplanted into the damaged spinal cord secrete a variety of neurotrophic factors in vivo by activating formant and damaged nerve cells, thus achieving neuroprotective functions, and 3 ) stem cells can form new nerve fibers, thereby promoting functional recovery of damage. However, the field of spinal cord regeneration and neural stem cell transplantation for the treatment of spinal cord injury is still in development and will require further study.

Biofilms (biological membrane) are inlaid with a glycoprotein phospholipid bilayer, which contains enzyme binding sites, whose function is to divide and separate cells and organelles, as well as a variety of energy conversion and intracellular communicational barrier functions. Research has shown that membrane damage is extensive and radicals, whose main role is to free or unsaturate fatty acid after the formation of lipid peroxide (LPO), therefore the content of LPO radical damage may reflect the degree of tissue cells (Trivedi et al., 2006; Lin et al., 2012; Chittiboina et al., 2012). SOD (superoxide dismutase) is the specificity of superoxide radical scavenging enzymes, it can significantly reduce free radical-mediated lipid peroxidation, lysosomal membrane stability, and thus the cell protective effect (Atılgan, 2012; Ouardouz et al., 2009; Hawryluk et al., 2008; Retraction, 2011; Paterniti et al., 2009; Liu et al., 2011). With MDA significantly increased after SCI, SOD significantly reduced, suggesting that lipid peroxidation in damage localization increased, it is also the result of increased free radicals (Gonzalez et al., 2003; Wang et al., 2009; Hakan et al., 2011). Free radicals and lipid peroxidation secondary damage after SCI plays and important role, and early application after spinal cord injury promoted radical scavengers, reducing the production of MDA increased SOD activity (Zhao et al., 2011; Kumar et al., 2011; Vinit, 2012; Krause et al., 2012). Blood flow changes after SCI, and extensive infiltration of inflammatory cells also result in a large amount of oxygen free radicals (Donnelly et al., 2008). After spinal cord injury, the major lipid peroxidation end product MDA and 4-Hydroxy Acrolein (4-HE) can be detected, indicating that lipid peroxidation is one of the pathological changes after spinal cord injury, experiments have proven to reduce lipid peroxidation after spinal cord injury to animals improving motor function (Trivedi et al., 2006; Paterniti et al., 2009; Retraction, 2011; Liu et al., 2011; Chittiboina et al., 2012). We also found that in the experiment, MDA levels in nimodipine group were significantly lower, indicating that nimodipine might play a role in anti-oxidation by scavenging free radicals. In addition, the nimodipine treatment spinal cord injury rats significantly decreased MPO activity, ED1-positive markers significantly reduced; whether nimodipine functions by inhibiting microglial activation and inflammatory leukocyte infiltration needs further study.

Our study shows that nimodipine treatment after spinal cord injury in rats can reduce free radical oxidative damage, reducing the void area and inflammatory cell infiltration, with the promotion of the repair of spinal cord injury, suggesting that early treatment with nimodip- 
ine spinal cord injury has a significant effect. Good results have been found in many reports (Wang et al., 2009; Cho et al., 2011; Cai et al., 2011). We believe that nimodipine is without toxic side effects, and is safe, reliable, and easy to use for clinical treatment of spinal cord injury.

\section{ACKNOWLEDGMENTS}

Research supported by the Science Foundation of 2012 Affiliated Hospital of Yanan University Foundation (\#2012YDFY-J304).

\section{REFERENCES}

Atılgan M (2012). Double-level spinal cord injury without vertebral fracture or dislocation: A case report. Ulus Travma Acil Cerrahi Derg 18: 80-82.

Cai YF, Fan R, Hua TM, Liu HL, et al. (2011). Nimodipine alleviates apoptosis-mediated impairments through the mitochondrial pathway after spinal cord injury. Curr. Zool. 57: 340-349.

Chittiboina P, Cuellar-Saenz H, Notarianni C, Cardenas R, et al. (2012). Head and spinal cord injury: diagnosis and management. Neurol. Clin. 30: 241-276.

Cho YA, Choi JS and Burm JP (2011) Effects of the antioxidant baicalein on the pharmacokinetics of nimodipine in rats: a possible role of P-glycoprotein and CYP3A4 inhibition by baicalein. Pharmacol. Rep. 63: 1066-1073.

Donnelly DJ and Popovich PG (2008). Inflammation and its role in neuroprotection, axonal regeneration and functional recovery after spinal cord injury. Exp. Neurol. 209: 378-388.

Doukas A, Petridis AK, Barth H, Jansen O, et al. (2011). Resistant vasospasm in subarachnoid hemorrhage treated with continuous intraarterial nimodipine infusion. Acta Neurochir. (Suppl 112): 93-96.

Gonzalez R, Glaser J, Liu MT, Lane TE, et al. (2003). Reducing inflammation decreases secondary degeneration and functional deficit after spinal cord injury. Exp. Neurol. 184: 456-463.

Gruner JA (1992). A monitored contusion model of spinal cord injury in the rat. J. Neurotrauma 9: 123-126.

Hakan T, Toklu HZ, Biber N, Celik H, et al. (2011). Meloxicam exerts neuroprotection on spinal cord trauma in rats. Int. J. Neurosci. 121: 142-148.

Han YY, Gui W, Zhu J, Wang K, et al. (2011). Thunderclap headache triggered by micturition: responsive to nimodipine. J. Headache Pain 12: 649-651.

Hawryluk GW, Rowland J, Kwon BK and Fehlings MG (2008). Protection and repair of the injured spinal cord: a review of completed, ongoing, and planned clinical trials for acute spinal cord injury. Neurosurg. Focus 25: E14.

Krause JS, Terza JV, Erten M, Focht KL, et al. (2012). Prediction of postinjury employment and percentage of time worked after spinal cord injury. Arch. Phys. Med. Rehabil. 93: 373-375.

Kumar R, Singh SS, Pranav and Ray SB. (2011). Nimodipine potentiates the analgesic effect of morphine in the rat hotplate test: Implications in the treatment of pain. Indian J. Anaesth. 55: 413-416.

Lang C, Guo X, Kerschensteiner M and Bareyre FM (2012). Single collateral reconstructions reveal distinct phases of corticospinal remodeling after spinal cord injury. PLoS One 7: e30461.

Lin MR, Yu WY and Wang SC (2012). Examination of assumptions in using time tradeoff and standard gamble utilities in individuals with spinal cord injury. Arch. Phys. Med. Rehabil. 93: 245-252.

Liu C, Shi Z, Fan L, Zhang C, et al. (2011). Resveratrol improves neuron protection and functional recovery in rat model of spinal cord injury. Brain. Res. 1374: 100-109.

Lockman JA, Geldenhuys WJ, Bohn KA, Desilva SF, et al. (2012). Differential effect of nimodipine in attenuating ironinduced toxicity in brain-and blood-brain barrier-associated cell types. Neurochem. Res. 37: 134-142.

Narazaki DK, Barros Filho TE, Oliveira CR, Cristante AF, et al. (2006). Spinal cord regeneration: the action of neurotrophin-3 in spinal cord injury in rats. Clinics 61: 453-460.

Ouardouz M, Coderre E, Basak A, Chen A, et al. (2009). Glutamate receptors on myelinated spinal cord axons: I. GluR6 kainate receptors. Ann. Neurol. 65: 151-159.

Paterniti I, Genovese T, Crisafulli C, Mazzon E, et al. (2009). Treatment with green tea extract attenuates secondary inflammatory response in an experimental model of spinal cord trauma. Naunyn Schmiedebergs Arch. Pharmacol. 380: $179-192$.

Retraction (2011). Activated protein C reduces the severity of compression-induced spinal cord injury in rats by inhibiting activation of leukocytes. J. Neurosci. 31: 8697. 
Saunders LL and Krause JS (2012). Behavioral factors related to fatigue among persons with spinal cord injury. Arch. Phys. Med. Rehabil. 93: 313-318.

Tan HC, Li JN and Bu L (2005). OECs transplantation combined with nimodipine promote functional recovery after spinal cord injury in rats. Zhong Guo Jiao Xing Wai Ke Za Zhi 13: 290-291.

Trivedi A, Olivas AD and Noble-Haeusslein LJ (2006). Inflammation and spinal cord injury: infiltrating leukocytes as determinants of injury and repair processes. Clin. Neurosci. Res. 6: 283-292.

Vinit S (2012). Cervical spinal cord injuries and respiratory insufficiency: a revolutionary treatment. Med. Sci. 28: 33-36.

Wang Y, Lapitsky Y, Kang CE and Shoichet MS (2009). Accelerated release of a sparingly soluble drug from an injectable hyaluronan-methylcellulose hydrogel. J. Control Release 140: 218-223.

Wijesuriya N, Tran Y, Middleton J and Craig A (2012). Impact of fatigue on the health-related quality of life in persons with spinal cord injury. Arch. Phys. Med. Rehabil. 93: 319-324.

Zhang L, Chen HB, Wang Y and Zhang LY, et al. (2012). Cervical spinal canal narrowing and cervical neurological injuries. Chin. J. Traumatol. 15: 36-41.

Zhao J, Zhou D, Guo J, Ren Z, et al. (2011). Efficacy and safety of fasudil in patients with subarachnoid hemorrhage: final results of a randomized trial of fasudil versus nimodipine. Neurol. Med. Chir. 51: 679-683.

Zhou TT and Zheng ZH (2008). Cord injury animal model construction and application. Shi Yan Dong Wu Yu Bi Jiao Yi Xиe 28: 411-415. 